\title{
Cranial Magnetic Resonance Imaging in Elderly American Indians: Design, Methods, and Implementation of the Cerebrovascular Disease and Its Consequences in American Indians Study
}

\author{
Astrid M. Suchy-Dicey ${ }^{a}$ Dean Shibata ${ }^{b}$ Lyle G. Best ${ }^{e}$ Steven P. Verney ${ }^{f}$ \\ William T. Longstreth, Jr. ${ }^{c}$ Elisa T. Lee ${ }^{g}$ Peter M. Okin ${ }^{\text {h }}$ Richard Devereux ${ }^{h}$ \\ Marcia O'Leary ${ }^{\mathrm{e}}$ Tauqeer Alig Paul N. Jensen ${ }^{\mathrm{a}}$ Clemma Muller $^{\mathrm{a}}$ \\ Lonnie A. Nelson $^{a}$ Everett Rhoades ${ }^{g}$ Tara Madhyastha $^{b}$ Thomas J. Grabowski ${ }^{b, c}$ \\ Norman Beauchamp ${ }^{b}$ Jason G. Umans ${ }^{d}$ Dedra Buchwald ${ }^{a}$

\begin{abstract}
a Partnerships for Native Health, Elson S. Floyd College of Medicine, Washington State University, and Departments of ${ }^{b}$ Radiology and ${ }^{\mathrm{C} N e u r o l o g y, ~ U n i v e r s i t y ~ o f ~ W a s h i n g t o n, ~ S e a t t l e, ~ W a s h ., ~}{ }^{\mathrm{d}}$ Georgetown-Howard Universities Center for Clinical and Translational Science, Washington, D.C., ' Missouri Breaks Industries Research, Inc., Eagle Butte, S.Dak., ${ }^{f}$ Department of Psychology, University of New Mexico, Albuquerque, N.Mex., ${ }^{9}$ Center for American Indian Health Research, College of Public Health, University of Oklahoma, Oklahoma City, Okla., and h Cardiovascular Reading Center, Weill Cornell Medical College, New York, N.Y., USA
\end{abstract}

\section{Key Words}

Vascular brain injury · Cognitive performance .

Cerebrovascular disease $\cdot$ Risk factors $\cdot$ American Indians

\begin{abstract}
The Cerebrovascular Disease and its Consequences in American Indians (CDCAI) Study recruited surviving members of a 20-year, longitudinal, population-based cohort of American Indians focused on cardiovascular disease, its risk factors, and its consequences. The goal of the CDCAI Study is to characterize the burden, risk factors, and manifestations of vascular brain injury identified on cranial MRI. The CDCAI Study investigators enrolled 1,033 participants aged 60 and older from 11 American Indian communities and tribes in the Northern Plains, Southern Plains, and Southwestern United States. In addition to cranial MRI performed according to standardized protocols, participants underwent extensive
\end{abstract}

medical interview, clinical examination, neurocognitive testing, physical function evaluation, electrocardiogram, and provided blood and urine specimens. Participants also selfadministered questionnaires covering demographics, quality of life, and medical history. This report describes the design, implementation, and some of the unique challenges of this study and data collection.

(c) 2016 S. Karger AG, Basel

Vascular brain injury can be clinically overt, as with stroke or transient ischemic attack, or clinically covert, in the absence of such events and defined by findings on imaging or pathological examination. Relative to other US populations, American Indians have a high prevalence and incidence of stroke [1-3] and a young mean age at stroke onset $[4,5]$. Among American Indians younger than 65 years, rates of stroke fatality are more than 3 times

\section{KARGER}

E-Mail karger@karger.com

www.karger.com/ned (c) 2016 S. Karger AG, Basel

0251-5350/16/0472-0067\$39.50/0
Astrid M. Suchy-Dicey, $\mathrm{PhD}$

Partnerships for Native Health

Washington State University

1100 Olive Way, Suite 1200 Seattle, WA 98101 (USA)

E-Mail astrid.suchy-dicey@wsu.edu 
higher than that of whites with similar age [1]. Despite such dramatic disparities, stroke and vascular brain injury remain understudied among American Indians, even by efforts specific to racial and ethnic disparities in stroke [6-9].

The source population for Cerebrovascular Disease and Its Consequences in American Indians (CDCAI) Study is, to date, the largest prospective cohort study among American Indians of cardiovascular disease, including stroke [10]. From 1989 to 1991, field staff enrolled 4,549 American Indians aged 45-74 from 13 communities in the Northern Plains, the Southern Plains, and the Southwestern United States [10]. Among the 4,507 original cohort participants without a history of stroke at baseline, 306 (6.8\%) sustained incident stroke at a mean age of 66.5 through 2008 [11]. The age- and gender-adjusted stroke incidence observed in this American Indian cohort - 6.8 per 1,000 person-years [11] - is more than twice that of the general US population [5], and higher than estimates for African Americans and whites from the Framingham study [12], the Atherosclerosis Risk in Communities (ARIC) study [13], and the Cardiovascular Health Study (CHS) [14].

The original cohort, the Strong Heart Study, collected information about stroke, but unlike other large population-based studies such as ARIC and CHS, it did not collect or examine measures of covert vascular brain injury, as defined by findings on MRI. Cranial MRI provides excellent contrast and spatial resolution, and is particularly sensitive to white matter hyperintensities (WMH), infarcts, hemorrhages, focal and regional brain atrophy, and intracranial mass lesions [15]. Covert vascular brain injury may be more prevalent than clinical disease; one study of elderly participants imaged twice over 5 years found that incident MRI-defined infarcts were more than 6 times more frequent than incident adjudicated stroke [16]. In addition, MRI findings of vascular brain injury in the elderly are associated with impairments in cognition, mobility, and mood as well as with risk of subsequent stroke, dementia, and death [17-19]. Using MRI to measure covert vascular brain injury in a large cohort of American Indians will enable characterization of a substantial public health concern in a population likely to be heavily affected.

The CDCAI Study, known to participants and communities as the Strong Heart Stroke Study, which commenced recruitment in 2009 and data collection in 2010, invited surviving original participants of a large epidemiological study on cardiovascular disease who were residing in the Northern Plains, the Southern Plains, and the Southwestern United States to undergo cranial MRI and related clinical, cognitive, and functional examination. The study's principal objectives were to estimate the prevalence and extent of vascular brain injury as defined by cranial MRI, among American Indians; examine associations with risk factors; and characterize disease manifestations, especially in relation to impairments in cognition, mobility, and mood. In this report, we describe the design and implementation of the study data collection, detailing the extensive participant examinations that were completed and describing some of the practical challenges to execution.

\section{Participant Recruitment}

CDCAI Study comprises a set of examinations of participants who were eligible for MRI examination between 2009 and 2013 (fig. 1) [10, 20]. Participants were excluded if they had (1) prior surgery for a cerebral aneurysm; (2) an implanted cardiac pacemaker, defibrillator, or artificial heart; (3) contraindicating metal prostheses; (4) a cochlear implant, spinal cord stimulator, or other internal electrical device; (5) history of employment as a metal worker (given the possibility of retained metal fragments, especially near the eyes); (6) weight of 350 pounds or more; or (7) physical or cognitive inability to complete study procedures. The first 6 criteria matched the MRI screening criteria used by CHS $[21,22]$ and ARIC [23, $24]$. The seventh criterion was based on a practical decision to take into account the elderly and often frail condition of potential participants, especially those in chronic care facilities.

Participants were recruited from 3 study sites in the Northern Plains, the Southern Plains, and the Southwestern United States, representing 11 of the original $13 \mathrm{com}$ munities [10]. Trained field staff made the initial contact - either by telephone or during a planned home visit, explained the purpose of the study, invited participation, and screened for eligibility. A recruitment target of 1,000 participants was established a priori, based on the expected number of potential participants and the expected prevalence of MRI findings. All study participants gave informed consent, and all study procedures were approved by 13 separate organizations, comprising 5 Tribal Review Boards or Tribal Councils, 5 academic or medical Institutional Review Boards (IRBs), and 3 regional Indian Health Service IRBs. The CDCAI Study's Coordinating Center is located at Partnerships for Native Health in Seattle Washington. Data collection was conducted through 2013.
Suchy-Dicey et al. 


\section{Magnetic Resonance Imaging}

After providing informed consent, participants attended a clinic visit for cranial MRI examination. Clinic technicians assessed scan eligibility a second time by following an established clinical protocol based on the first 6 eligibility criteria. Contrast agent was not used. Scan protocols were optimized for interpretation and quantitative analysis, compatibility with the level of technology at the regional hospitals where the scans were performed, consistency across study sites and scanners, consideration of time constraints and endurance of elderly participants, and compatibility with study procedures used by CHS and ARIC [21, 22, 24, 25]. The scanners were Siemens 1.5T Symphony (Siemens Medical Solutions, Malvern, Pa., USA) in the Northern Plains (RCMRI) and General Electric 1.5T Signa (General Electric Healthcare, Little Chalfont Buckinghamshire, UK) in the Southern Plains (GEMSOW) and Southwest (MR1, MR2, and MR3). Sagittal and axial T1 and T2 sequences and machine parameters were standardized as much as possible across the 5 individual machines.

Neuroradiologists trained in the study protocols and blinded to participant age, gender, and clinical information read and graded all MRI scans. A primary reader scored all aspects of the scan images, including perivascular space, infarcts, hemorrhages, $\mathrm{WMH}$, sulci, and ventricles; a secondary reader assessed brain infarcts and hemorrhages. Scans for which the 2 readers returned discrepant scores were reviewed until consensus was reached. Infarcts and hemorrhages were scored by number, size, type, and location. The severity of WMH, sulcal enlargement, and ventricular enlargement were graded on a 10 -point scale [21]. In addition to the graded measures, quantitative volumes were estimated using automated software, including FLEX for WMH [26]; FIRST in FSL 5.0 and the ENIGMA1 protocol for the hippocampus [27-29]; and FreeSurfer for total brain (white and grey matter) and intracranial space [30].

\section{Physical and Cognitive Examinations}

Participants also underwent a series of physical examinations and neurocognitive testing at an additional clinic visit completed within 30 days of the MRI scan. Clinic and neurocognitive test protocols were designed to be brief to accommodate the older, often frail condition of participants, as well as to maximize comparability with existing cohort studies [31-33]. Participants

Cranial MRI in Elderly American Indians were asked to fast for $12 \mathrm{~h}$ before the visit. During the physical examination, anthropometric data were collected by following standardized protocols, including measurement of height, measurement of weight with the Tanita BWB-8005 adult digital scale (Tanita Corporation of America, Arlington Heights, Ill., USA), and measurement of supine waist girth, erect hip circumference, and upper arm circumference by using Novel Products Figure Finder tape measure (Novel Products, Rockton, Ill., USA). Sitting blood pressure was measured 3 times by using an Omron HEM-907 oscillometric blood pressure device (Omron Healthcare Inc., Lake Forest, Ill., USA), and then averaged. Performancebased tests were administered, including timed standing, timed walk, grip strength, and chair stands. Evaluations of cognitive function used the 100-point Modified Mini-Mental State [34]; Wechsler Adult Intelligence Scale coding test [35]; California Verbal Learning Test, second edition (Short Form) [36, 37]; Halstead finger tapping test [38]; and Controlled Oral Word Association test $[39,40]$.

\section{Administered Interviews}

At the clinic visit, trained field staff interviewed participants to collect demographics, health behaviors, and medical history. Specific data collected include gender, age, marital status, education, and income; details on smoking and alcohol use; prior diagnoses of high blood pressure, diabetes, heart disease, heart-related procedures, stroke and TIA; detailed history of symptoms related to stroke and TIA; detailed neurological history including Alzheimer's disease, Parkinson's disease, dementia, seizures, headaches, and head trauma. Medication use was also recorded by using the transcription of labels from medication bottles, which participants were asked to bring with them.

\section{Self-Report Questionnaires}

Participants also completed self-administered questionnaires, which collected information on personal health attitudes and beliefs; daily physical activity; physical pain; emotional health; history of trauma; family medical history; use of tribal language [41]; quality of life, as measured by the Short Form-36 [42]; and symptoms of depression as measured by the Centers for Epidemiologic Studies Depression Scale [43]. 


\section{Electrocardiogram}

A 12-lead resting digital electrocardiogram (ECG) was recorded at the clinic visit using the Marquette Mac-12 digital system (GE-Marquette Medical Systems, Milwaukee, Wisc., USA) [44]. For each ECG, $10 \mathrm{~s}$ of data were digitally recorded at a $500-\mathrm{Hz}$ sampling frequency to a resolution of $5 \mu \mathrm{V}$ and stored in a Marquette MUSE system for measurement and Minnesota code determination. ECGs were electronically transmitted from the clinic to the New York Presbyterian HospitalWeill Cornell Medical Center, where they were read by a single board-certified cardiologist (P.M.O.) who was blinded to participant age, gender, and clinical information.

\section{Laboratory Assays}

Participants provided fasting urine and serum samples at the clinic visit, which were immediately aliquoted into serum, EDTA plasma, NaF-EDTA plasma, Na-citrate plasma, whole blood, buffy coat, and urine. Aliquots were frozen at $-80^{\circ} \mathrm{C}$ and shipped to Penn Medical Laboratory (Hyattsville, Md., USA) for storage and assay. Assays were chosen based on known determinants and manifestations of cardiovascular disease and stroke to maximize comparability with related cohort studies [10, 31, 33]. Total cholesterol, HDL-cholesterol, LDL-cholesterol, and triglycerides were measured in serum on the Vitros 5.1 platform (Ortho Clinical Diagnostics, Rochester, N.Y., USA) using methods traceable to the National Reference System for Cholesterol by the Cholesterol Reference Method Laboratory Network. Glucose was assayed in NaF-plasma by a glucose oxidase method on the Vitros platform. Urine albumin was assayed by immunoturbidimetric methods, and creatinine was assayed by enzymatic rate methods, both on the $\mathrm{Vi}$ tros platform. High-sensitivity C-reactive protein and cystatin $\mathrm{C}$ were batch-assayed in stored serum samples by using reagents from Ortho Clinical Diagnostics or Gentian AS (Moss, Norway). Hemoglobin A1C was assayed from stored whole blood for participants with fasting plasma glucose $>110 \mathrm{mg} / \mathrm{dl}$ by using high-performance liquid chromatography (Tosoh Bioscience, South San Francisco, Calif., USA) traceable to National Glycohemoglobin Standardization Program standards. Quality control was implemented through continuous review. The College of American Pathologists certified the assay laboratory.

\section{Quality Assurance and Training}

A quality control committee oversaw the conduct and evaluation of study procedures. Field staff were responsible for reviewing data as they became available and resolving any quality control problems detected. Quality assurance measures also included quarterly observation and evaluation of data by field staff managers. Re-training was provided as needed.

Field staff, predominantly long-time residents of the same communities or tribes as study participants, were trained in standardized protocols for eligibility screening; obtaining consent; administering questionnaires and interviews; administering cognitive and physical performance tests; collecting anthropometric and blood pressure data; and collecting and processing biological samples. Their training included instructions on building rapport; facilitating a comfortable, confidential, and cooperative environment; and completing standard forms. Training specific to interviewing included adhering to standardized question protocols, maintaining a nonjudgmental attitude, appropriate prompting, appropriate problem-solving, proper handling of participant comments, documenting relevant information, and handling post-interview responsibilities.

Quality control of MRI data included testing and demonstration at each MRI clinic for expected image quality and adherence to study protocols. Professional clinical psychologists trained field staff in cognitive assessment procedures, and participant assessments were reviewed for test scoring. Field staff maintained all study equipment, including scales, tape measures, blood pressure monitors and cuffs, and ECG apparatus. Coordinating Center staff monitored ECGs on a quarterly basis to ensure accurate and consistent examinations. For laboratory assays, duplicate blood and urine specimens were collected from approximately $5 \%$ of the participants, assigned unique identifiers, and sent for blinded assay; results for duplicates were analyzed by the Coordinating Center for accuracy and consistency.

Field staff checked data forms manually for completeness, and duplicate entries were used to identify manual entry errors. Data entry programs added automatic data range and logic checks. Histograms, frequencies, and cross-tabulations were used to identify outliers as potentially erroneous values. Summary statistics, such as mean, median, range, maximum and minimum for continuous variables, and frequency distributions for categorical variables, were calculated regularly for each study site. Inconsistent data were flagged for review.
Suchy-Dicey et al. 


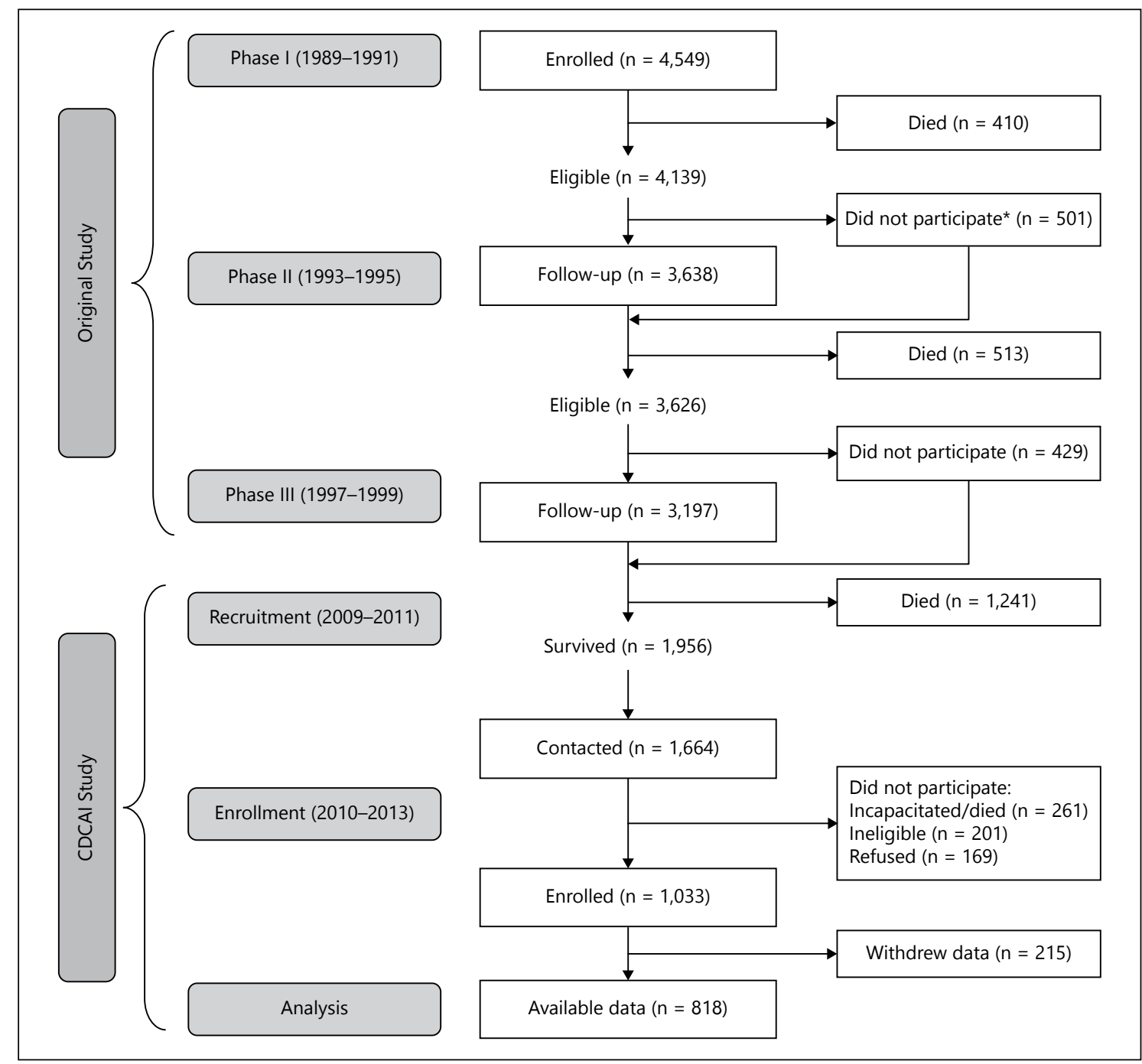

Fig. 1. Flow diagram of participants recruited from the original cohort and recruited into the CDCAI Study. * Those who did not participate in Phase II were invited to participate in Phase III.

\section{Participant Referral for Incidental Findings}

During the clinical examination, participants received educational materials on the importance of reducing risk factors and adopting healthy lifestyle habits. Participants were informed of their blood pressure, weight, and any abnormal or incidental findings observed during evaluation. Participants with abnormal findings were referred for additional care according to established guidelines for emergency, immediate, urgent, and routine referrals. Local radiologists at each hospital where MRI scans were performed screened images for evidence of abnormalities such as aneurysms, tumors, acute hemorrhage, and infarcts larger than $4 \mathrm{~cm}$. Any incidental findings prompted

Cranial MRI in Elderly American Indians a standard response that included immediate participant notification, a telephone call to the participant's primary care provider, and a brief letter summarizing the general category of the abnormality. Assistance was also provided to arrange consultations or referrals to health providers on the basis of participants' needs.

\section{Study Population}

Of the 1,956 members of the original cohort alive at the time of CDCAI Study recruitment, 1,664 were successfully contacted, but 261 subsequently died or became incapacitated before they could enroll (fig. 1). Of the 1,403 
contacted and available, 201 were ineligible and 169 declined, leaving a final CDCAI Study population of 1,033 and successful recruitment of $86 \%$ of eligible cohort members. After examinations were completed, one tribe selected on behalf of its 215 tribal members to decline participation in future analyses.

The study population $(\mathrm{n}=818)$ was predominantly elderly and female, with approximately comparable recruitment among the 3 study locations (table 1). Few participants were never married (5\%), and many were widowed $(33 \%)$. Most graduated from high school or attended at least some college (66\%), but income was generally low, with about one-third (30\%) living below the poverty line for one-person or 2-person households including members older than 65 years [45].

Although every effort was made in all cases to collect complete information, some missing data were unavoidable (table 2). The major reasons for missing data included technical errors or data corruption (ECG transmission errors, lab sample contamination, or MRI image quality concerns), and participant refusal or no-show.

Study recruitment from a 20-year cohort of elders posed questions about selection. We assessed selection comparing the original cohort of participants who did not enroll in CDCAI Study $(\mathrm{n}=3,482)$ with those who eventually did $(\mathrm{n}=1,033)$. The 3 sites were evenly distributed, with minor differences of 3 or $4 \%$. Age at original baseline examination (1989-1991) did differ significantly ( $\mathrm{p}<$ $0.001)$, with non-enrollees a mean of 6 years older $(58, \mathrm{SD}$ 8) than eventual enrollees (52, SD 6). Gender also differed significantly ( $\mathrm{p}<0.001$ ), with greater proportion of females among eventual enrollees (69\%) than non-enrollees $(57 \%)$. These differences reflect the imbalance in expectation of survival for different participants, and should be addressed in future reports with full analysis on survival and development of statistical methods to account for it.

\section{Potential Implications of Study Data}

This is the first study to conduct cranial MRI in elderly American Indians. Future research using these data will include investigation of the prevalence, risk factors, and manifestations of vascular brain injury in this population, with particular focus on subclinical disease. The use of MRI findings to study vascular brain injury will allow investigators to develop more thorough epidemiological, pathological, and clinical understanding of stroke and
Table 1. Participant characteristics $(n=818)$

\begin{tabular}{|c|c|}
\hline Age at clinic exam, mean (SD) & $73.0(5.9)$ \\
\hline Female, $\mathrm{n}(\%)$ & $555(67.9)$ \\
\hline \multicolumn{2}{|l|}{ Site, $\mathrm{n}(\%)$} \\
\hline Northern plains & $374(45.7)$ \\
\hline Southern plains & $346(42.3)$ \\
\hline Southwest & $98(12.0)$ \\
\hline \multicolumn{2}{|l|}{ Marital status, n (\%) } \\
\hline Never married & $43(5.3)$ \\
\hline Married/partnered & $310(37.9)$ \\
\hline Divorced/separated & $192(23.5)$ \\
\hline Widowed & $273(33.4)$ \\
\hline \multicolumn{2}{|l|}{ Education, $\mathrm{n}(\%)$} \\
\hline Up to or any high school & $162(19.8)$ \\
\hline Graduated high school & $210(25.7)$ \\
\hline Any college & $322(39.4)$ \\
\hline College graduate and beyond & $124(15.2)$ \\
\hline \multicolumn{2}{|l|}{ Income, n (\%), /year } \\
\hline$<\$ 10,000$ & $251(30.7)$ \\
\hline$\$ 10-20,000$ & $234(28.6)$ \\
\hline$\$ 20-35,000$ & $175(21.4)$ \\
\hline$>\$ 35,000$ & $158(19.3)$ \\
\hline \multicolumn{2}{|c|}{ Native language speaking capacity, n (\%) } \\
\hline Not at all & $262(32.1)$ \\
\hline A little & $235(28.8)$ \\
\hline Moderately & $101(12.4)$ \\
\hline Very well & $219(26.8)$ \\
\hline \multicolumn{2}{|l|}{ Language spoken at home, n (\%) } \\
\hline Native language only & $36(4.4)$ \\
\hline Native language and English & $235(28.8)$ \\
\hline English only & $546(66.8)$ \\
\hline
\end{tabular}

Table 2. Reasons for missing data $(n=1,033)$

$\begin{array}{lc}\text { ECG missing, n (\%) } & 29(2.8) \\ \text { Technical/transmission errors } & \text { All } \\ \text { Lab assays missing, n (\%) } & 7(0.6) \\ \text { Sample contamination } & 4 \\ \text { Refusal of blood draw } & 2 \\ \text { Inability to draw blood } & 1 \\ \text { Neurocognitive tests missing, n (\%) } & 3(0.3) \\ \text { Incomplete clinical exam } & \text { All } \\ \text { MRI missing, n (\%) } & 35(3.4) \\ \text { Deemed ineligible at clinic visit } & 22 \\ \text { Excess motion/poor image quality } & 9 \\ \text { Image corruption/data loss } & 4 \\ \text { Withdrew data after examination, n (\%) } & 215(20.8)\end{array}$

vascular brain injury, with the ultimate goal of developing appropriate interventions.

Because CDCAI Study participants were members of the original cohort, which represents a well-defined, geographically diverse population of American Indians at
Suchy-Dicey et al. 
particularly high risk of clinical stroke, future analyses will additionally benefit from potential for combination of these data with more than 20 years of prospectively collected data (1989-2010).

\section{Successes of Study Operations}

This large epidemiologic study required extensive coordination of complicated examination protocols in diverse and often remote geographic areas. However, the recruitment rate for the current study (86\%) was high, considering that this cohort consists entirely of elderly members of a racial minority; recruitment and retention rates for aging minority cohorts in other studies range from 60 to $80 \%$ [10,46-48]. The robust existing research infrastructure of original cohort sites and field clinics, as well as the dedication of field staff were fundamental to successful study implementation. Study coordinators and clinical personnel have longstanding rapport with cohort participants and a strong working relationship with local tribal councils and leadership. Field staff were long-time residents of the same communities or tribes as study participants, making them particularly mindful of cultural concerns among older community members. In addition, field staff were trained in methods for interviewing and interacting with elderly patients, imparting capacity to engage in successful, sensitive communication with participants at all stages of study execution. Such accommodations facilitated the inclusion of cohort members who might otherwise have been disinclined to participate. In addition, study investigators and coordinating staff regarded tribal representatives as key partners in the research enterprise, providing regular, in-person updates on study progress and protocol changes to these critical stakeholders. Regularly consultations with tribes and attendance at tribal meetings promoted trust among tribal officials and study participants, who often express pride in their membership.

\section{Challenges of Study Operations}

Despite the successes, epidemiologic studies including rural, elderly American Indian participants still face particular challenges. The remoteness of some MRI clinic locations was problematic in rural sites, because research staff and participants were sometimes required to travel long distances at substantial cost to complete study procedures. Efforts were made to offset travel costs, but in some cases the long distances between participant residence and the nearest MRI facility limited recruitment. For example, in the Northern Plains, distances between field clinics (a proxy for participant residence) and study MRI facilities ranged from 90 to 180 miles, while in the Southern Plains and Southwest, distances were somewhat shorter, between 10 and 86 miles. Eligible participants who were unable to travel these distances were not enrolled. A small number of eligible participants residing in one community in the Northern Plains did not participate because their location was nearly 500 miles from the nearest study MRI facility; similarly, residents of a small Southwest community were not recruited because fewer than 5 surviving participants were eligible for enrollment, making coordination with local MRI facilities untenable. Inclement weather added to the difficulties of travel in some areas, both at the exam facilities and in participants' home communities. Close monitoring of weather forecasts and careful planning were often required, especially during winter in the Northern Plains.

The advanced age of the study population also required special consideration, so MRI and clinic exam study protocols were designed specifically to accommodate the needs of the elderly, especially with regard to duration. Field staff transported participants between their homes and the nearest MRI facility, but commute times were still sometimes in excess of $3 \mathrm{~h}$ each way. MRI scan protocols were limited to allow for limited participant endurance as well as limited ability of some elderly to remain perfectly still.

\section{Study Limitations}

This study has certain limitations. Participant survival between the selection of the original cohort and the MRI examinations were low, so this study represents a population of survivors. Therefore, descriptive analyses should be generalized with caution and inference-oriented analyses should use inverse probability weighting to evaluate the possibility of selection bias. Standardized methods specific to this cohort are in development and will be reported separately. In addition, this study includes elder American Indians from the Northern Plains, Southern Plains, and Southwest United States; findings may not be generalizable to other populations. One tribal group declined participation on behalf of 215 tribal members after completion of data collection but before any research reports were published, leaving an available analytic study population of 818 . 
Relevant to imaging, cranial MRI is sensitive to technical errors and motion artifact; if the elderly participants prone to motion also comprised those with the most extreme disease, the detectability of MRI findings of vascular brain injury may be attenuated. Cranial MRI also does not represent direct pathologic examination of lesions, and atrophy cannot be directly observed without longitudinal evaluations, and so measurement error may occur. Detailed description and evaluation of MRI examinations is under development and will be reported separately.

\section{Research Using CDCAI Study Data}

In sum, the CDCAI Study collection of cranial MRI and related examinations will enable researchers to characterize vascular brain injury in a unique, high-risk population. Future research using these data may lead to enhanced understanding of epidemiology, etiology, pathol- ogy, and manifestations related to stroke and vascular brain disease. Interested researchers may contact Partnerships for Native Health, now at Washington State University, or the authors for more information about manuscript and data use procedures.

\section{Acknowledgments}

This work was supported by funding from the National Heart Lung and Blood Institute, HL093086 (D. Buchwald), HL065520 (Cole), and HL041654 (E.T. Lee).

Thanh Ton, $\mathrm{PhD}$ and Raymond Harris, $\mathrm{PhD}$ contributed to the drafting of this manuscript, and Andrea Krinke contributed to early research coordination efforts. We wish to thank all study participants.

\section{Disclosure Statement}

The authors do not have any financial conflicts to disclose.

\section{References}

1 Harwell TS, Oser CS, Okon NJ, et al: Defining disparities in cardiovascular disease for American Indians: trends in heart disease and stroke mortality among American Indians and whites in Montana, 1991 to 2000. Circulation 2005;112:2263-2267.

2 Rosamond W, Flegal K, Friday G, et al: Heart disease and stroke statistics - 2007 update: a report from the American Heart Association Statistics Committee and Stroke Statistics Subcommittee. Circulation 2007;115:e69-e171.

3 Stansbury JP, Jia H, Williams LS, et al: Ethnic disparities in stroke: epidemiology, acute care, and postacute outcomes. Stroke 2005;36: 374-386.

4 Go AS, Mozaffarian D, Roger VL, et al: Heart disease and stroke statistics - 2013 update: a report from the American Heart Association. Circulation 2013;127:e6-e245.

5 Centers for Disease Control and Prevention (CDC): Prevalence of stroke - United States, 2005. MMWR Morb Mortal Wkly Rep 2007; 56:469-474.

6 McGruder HF, Malarcher AM, Antoine TL, et al: Racial and ethnic disparities in cardiovascular risk factors among stroke survivors: United States 1999 to 2001. Stroke 2004;35: 1557-1561.

7 Schwamm LH, Reeves MJ, Pan W, et al: Race/ ethnicity, quality of care, and outcomes in ischemic stroke. Circulation 2010;121:14921501.

8 Cruz-Flores S, Rabinstein A, Biller J, et al: Racial-ethnic disparities in stroke care: the American experience: a statement for healthcare professionals from the American Heart
Association/American Stroke Association. Stroke 2011;42:2091-2116.

9 Roger VL, Go AS, Lloyd-Jones DM, et al: Heart disease and stroke statistics - 2012 update: a report from the American Heart Association. Circulation 2012;125:e2-e220.

10 Lee ET, Welty TK, Fabsitz R, et al: The strong heart study. A study of cardiovascular disease in American Indians: design and methods. Am J Epidemiol 1990;132:1141-1155.

11 Zhang Y, Galloway JM, Welty TK, et al: Incidence and risk factors for stroke in American Indians: the strong heart study. Circulation 2008;118:1577-1584.

12 Petrea RE, Beiser AS, Seshadri S, et al: Gender differences in stroke incidence and poststroke disability in the Framingham heart study. Stroke 2009;40:1032-1037.

13 Rosamond WD, Folsom AR, Chambless LE, et al: Stroke incidence and survival among middle-aged adults: 9-year follow-up of the atherosclerosis risk in communities (ARIC) cohort. Stroke 1999;30:736-743.

14 Arnold AM, Psaty BM, Kuller LH, et al: Incidence of cardiovascular disease in older Americans: the cardiovascular health study. J Am Geriatr Soc 2005;53:211-218.

15 Broderick DF: Neuroimaging in neuropsychiatry. Psychiatr Clin North Am 2005;28: 549-566, 64 .

16 Longstreth WT Jr, Dulberg C, Manolio TA, et al: Incidence, manifestations, and predictors of brain infarcts defined by serial cranial magnetic resonance imaging in the elderly: the cardiovascular health study. Stroke 2002;33: 2376-2382.
17 Vermeer SE, Longstreth WT Jr, Koudstaal PJ Silent brain infarcts: a systematic review. Lancet Neurol 2007;6:611-619.

18 Debette S, Markus HS: The clinical importance of white matter hyperintensities on brain magnetic resonance imaging: systematic review and meta-analysis. BMJ 2010;341:c3666.

19 Windham BG, Griswold ME, Shibata D, et al: Covert neurological symptoms associated with silent infarcts from midlife to older age: the atherosclerosis risk in communities study. Stroke 2012;43:1218-1223.

20 Rhoades DA, Welty TK, Wang W, et al: Aging and the prevalence of cardiovascular disease risk factors in older American Indians: the strong heart study. J Am Geriatr Soc 2007;55: 87-94.

21 Manolio TA, Kronmal RA, Burke GL, et al: Magnetic resonance abnormalities and cardiovascular disease in older adults. The cardiovascular health study. Stroke 1994;25:318-327.

22 Bryan RN, Manolio TA, Schertz LD, et al: A method for using MR to evaluate the effects of cardiovascular disease on the brain: the cardiovascular health study. AJNR Am J Neuroradiol 1994;15:1625-1633.

23 Bryan RN, Cai J, Burke G, et al: Prevalence and anatomic characteristics of infarct-like lesions on MR images of middle-aged adults: the atherosclerosis risk in communities study. AJNR Am J Neuroradiol 1999;20:1273-1280.

24 Liao D, Cooper L, Cai J, et al: Presence and severity of cerebral white matter lesions and hypertension, its treatment, and its control. The ARIC study. Atherosclerosis risk in communities study. Stroke 1996;27:2262-2270. 
25 Knopman DS, Penman AD, Catellier DJ, et al: Vascular risk factors and longitudinal changes on brain MRI: the ARIC study. Neurology 2011;76:1879-1885.

26 Gibson E, Gao F, Black SE, et al: Automatic segmentation of white matter hyperintensities in the elderly using FLAIR images at 3T. J Magn Reson Imaging 2010;31:1311-1322.

27 Stein JL, Medland SE, Vasquez AA, et al: Identification of common variants associated with human hippocampal and intracranial volumes. Nat Genet 2012;44:552-561.

28 Zhang Y, Brady M, Smith S: Segmentation of brain MR images through a hidden Markov random field model and the expectationmaximization algorithm. IEEE Trans Med Imaging 2001;20:45-57.

29 Patenaude B, Smith SM, Kennedy DN, et al: A Bayesian model of shape and appearance for subcortical brain segmentation. NeuroImage 2011;56:907-922.

30 Reuter M, Schmansky NJ, Rosas HD, et al: Within-subject template estimation for unbiased longitudinal image analysis. NeuroImage 2012;61:1402-1418.

31 The atherosclerosis risk in communities (ARIC) study: design and objectives. The ARIC investigators. Am J Epidemiol 1989; 129:687-702.

32 Mosley TH Jr, Knopman DS, Catellier DJ, et al: Cerebral MRI findings and cognitive func- tioning: the atherosclerosis risk in communities study. Neurology 2005;64:2056-2062.

33 Mittelmark MB, Psaty BM, Rautaharju PM, et al: Prevalence of cardiovascular diseases among older adults. The cardiovascular health study. Am J Epidemiol 1993;137:311-317.

34 Teng EL, Chui HC: The modified mini-mental state (3MS) examination. J Clin Psychiatry 1987;48:314-318.

35 Wechsler D: Wechsler Adult Intelligence Scale, ed 4. San Antonio, Pearson, 2008.

36 Lacritz LH, Cullum CM: The Hopkins verbal learning test and CVLT: a preliminary comparison. Arch Clin Neuropsychol 1998;13: 623-628.

37 Delis DC, Kramer JH, Kaplan E, Ober BA California Verbal Learning Test - Second Edition (CVLT-II). The Psychological Corporation, 2000.

38 Halstead W: Brain and Intelligence. Chicago: University of Chicago Press, 1947.

39 Ruff RM, Light RH, Parker SB, et al: Benton controlled oral word association test: reliability and updated norms. Arch Clin Neuropsychol 1996;11:329-338.

40 Benton AL, Hamsher K: Multilingual Aphasia Examination. Iowa City, AJA Associates, 1976.

41 Slattery ML, Schumacher MC, Lanier AP, et al: A prospective cohort of American Indian and Alaska Native people: study design, methods, and implementation. Am J Epidemiol 2007;166:606-615.

42 Ware JE Jr, Sherbourne CD: The MOS 36item short-form health survey (SF-36). I. Conceptual framework and item selection. Med Care 1992;30:473-483.

43 Radloff LS: The CES-D Scale: a self-report depression scale for research in the general population. Appl Psychol Meas 1977;1:385-401.

44 Okin PM, Roman MJ, Lee ET, et al: Combined echocardiographic left ventricular hypertrophy and electrocardiographic ST depression improve prediction of mortality in American Indians: the strong heart study. Hypertension 2004;43:769-774.

45 Census US: www.census.gov. 2010 (accessed January 12, 2014).

46 Ofstedal MB, Weir DR: Recruitment and retention of minority participants in the health and retirement study. Gerontologist 2011; 51(suppl 1):S8-S20.

47 Ejiogu N, Norbeck JH, Mason MA, et al: Recruitment and retention strategies for minority or poor clinical research participants: lessons from the Healthy Aging in Neighborhoods of Diversity across the Life Span study. Gerontologist 2011;51(suppl 1):S33-S45.

48 Hinton L, Carter K, Reed BR, et al: Recruitment of a community-based cohort for research on diversity and risk of dementia. Alzheimer Dis Assoc Disord 2010;24:234-241. 